\title{
A locally linear method for enforcing temporal smoothness in serial image registration
}

\author{
Ernst Schwartz ${ }^{1,2}$, Andras Jakab ${ }^{1,2}$, Gregor Kasprian ${ }^{2}$, \\ Lilla Zöllei ${ }^{3}$, and Georg Langs ${ }^{1,2}$ \\ 1 CIR Lab, Department of Biomedical Imaging and Image-guided Therapy, \\ ernst.schwartz@meduniwien.ac.at* \\ 2 Department of Biomedical Imaging and Image-guided Therapy \\ Medical University of Vienna \\ 3 Laboratory for Computational Neuroimaging, Martinos Center for Biomedical \\ Imaging, Massachusetts General Hospital
}

\begin{abstract}
Deformation fields obtained from image registration are commonly used for deriving measurements of morphological changes between reference and follow-up images. As the underlying image matching problem is ill-posed, the exact shape of these deformation fields is often dependent on the regularization method. In longitudinal and cross-sectional studies this effect is amplified if time between acquisitions varies and smoothness between serial deformations is neglected. Existing solutions suffer from high computational costs, strong modeling assumptions and the bias towards a single reference image. In this paper, we propose a computationally efficient solution to this problem via a temporal smoothing formulation in the one-parameter subgroup of diffeomorphisms parametrized by stationary velocity fields. When applied to modeling fetal brain development, the proposed regularization results in smooth deformation fields over time and high data fidelity.
\end{abstract}

\section{Introduction}

Accurately estimating the deformation of an anatomical structure as a function of time or age from a set of examples is central to the analysis of developmental [1] and degenerative processes [2]. Finding a temporal deformation field that represents the underlying process well, and at the same time captures the variability in the training population requires regularization.

Modelling fetal brain development $[3,4]$ is particularly challenging. The regularization method has substantial impact on the resulting deformation due to substantial morphological differences during short periods of time, partially homogeneous image regions, and smooth surfaces that lack gradient information. This limits the applicability of established motion estimation methods [5] and

* This research was supported by the Austian National Bank (14812, FETALMORPHO), the Austrian Science Fund (P 22578-B19, PULMARCH), and the European Union (FP7-ICT-2009-5/257528, KHRESMOI and FP7-ICT-2009-5/318068, VISCERAL) 
uniform priors during regularization. In this paper we propose a locally linear regularization that allows for spatial weighting of the temporal smoothing of resulting deformations.

The availability of a number of consecutive observations enables various solutions to the aperture problem. The rich mathematical formalism of diffeomorphism groups [6] provides a basis for many of these. For example, a theory of linear least-squares geodesic regression using the initial-momentum representation of diffeomorphisms [7] has been proposed in [8-10]. The authors in [11] use a vector momenta formulation to the same aim, while optimization of an acceleration-based model has been proposed in [12] in order to obtain smooth deformations between consecutive observations. The time-varying velocity field representation of diffeomorphisms [13] has been used for higher-order formulations such as spline interpolation [14] and kernel regression [15,16]. Adding a temporal component to the registration problem by smoothing the deformations to an explicit $[17,18]$ or implicit [19] common reference frame has also been proposed. Due to their efficient computation and useful mathematical properties, diffeomorphisms generated by Stationary Velocity Fields (SVF) [20] are commonly used in this setting. With the notable exception of [21], previously proposed regression and smoothing approaches rely on defining a common reference space for their operation. This can become increasingly difficult in the presence of large deformations, where regularization necessary to render the registrations tractable further shapes the resulting deformation fields. Also, the assumption of linearity can prove too strong in these cases. While theoretically established in a general sense [22], computation of higher-order models has so far only been applied to lower-dimensional shape representations such currents [23], which require a-priori segmentation of the structures of interst.

In [24], Lorenzi et al. proposed a method for estimating smooth longitudinal deformations using regression of pair-wise registrations encoded using SVFs. This requires a common reference space for all deformations and thus registration of a baseline to all later time-points. Instead, we propose to only consider a local temporal neighborhood for the temporal smoothing of the resulting deformations. This also enables us to increase the performance of our method by defining a local weighting of the temporal smoothing.

We briefly review the foundations of image registration in the log-euclidean framework for diffeomorphisms [20] in Section 2. We then show how its mathematical properties can be used to construct a spatially adaptive smoothing prior for serial image registration. We evaluate our method on a simplified synthetic model of cortical folding and a publicly available data set of fetal brain development [25] in Section 3.

\section{Method}

Let $S_{t_{n}}(x)$ be a set of $N$ observations of a continuous process affecting the shape of the observation space $\Omega$ at time points $t_{n}$ with $n=1, \ldots, N$. As the group of diffeomorphisms is closed under composition, we can obtain a diffeomorphic 
model of the overall deformation between time-points $t_{1}$ and $t_{N}$ by concatenating pair-wise diffeomorphic registrations $\phi_{n}$, so that $S_{t_{n}}\left(\phi_{n}(x)\right) \approx S_{t_{n+1}}(x)$. A mapping from any image $S_{i}$ to another $S_{j}$ with $j>i$ can be expressed as a concatenation of transforms $\Phi_{i j}=\phi_{i} \circ \cdots \circ \phi_{j}$ (Figure 1). Each $\phi_{j}$ is calculated based on an image pair and a prior on the deformation field that acts as regularization. Our aim is to find a spatio-temporal regularization prior that accurately models the deformation over time.

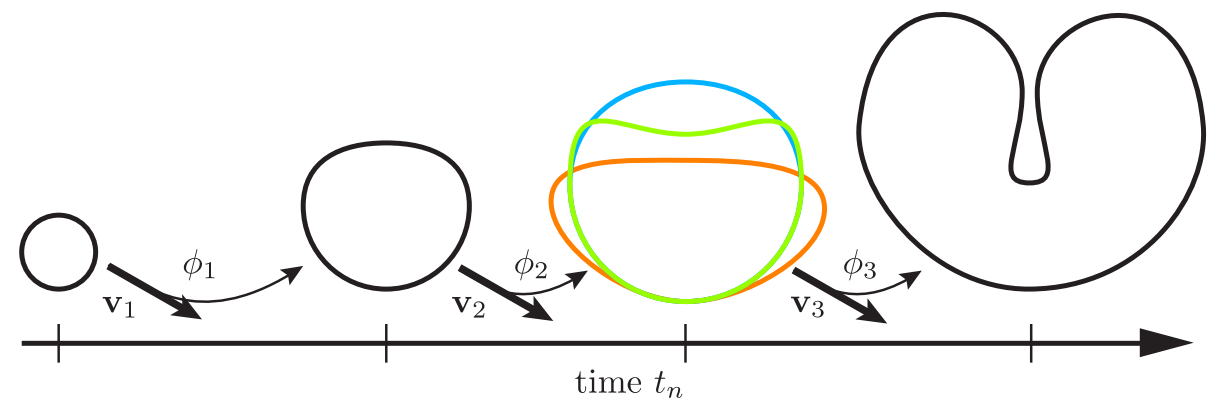

Fig. 1: Sketch of a continuous deformation and an intermediate shape between $t_{2}$ and $t_{3}$ computed from three possible registrations $\phi_{2}$

The properties of the SVF parametrization of diffeomorphisms can be exploited to formulate a prior on temporal smoothness in a chain of pairwise image registrations. Also, it enables to use the model residual as a weighting function that reduces the influence of the prior in regions where the assumption of constant deformation does not hold.

\subsection{Pair-wise LogDemons registration}

We calculate diffeomorphic mappings $\phi_{n}$ between images using the LogDemons algorithm [26]. The algorithm computes a diffeomorphism $\phi(x)$ defined on $x \in$ $\Omega \subset \mathbb{R}^{d}, d \in\{2,3\}$ and parametrized by an SVF $\mathbf{v}$ via the ODE

$$
\frac{d \phi(x, \tau)}{d \tau}=\mathbf{v}(\phi(x, \tau)), \quad \phi(x, 0)=\mathrm{id}
$$

Equation (1) represents a geodesic curve between a source image $S(x)$ and a target image $T \approx S(\phi(x, 1))$ in the one-parameter subgroup generated by SVF of the Lie group of diffeomorphisms $D$. The velocity field $\mathbf{v}$ is an element of the tangent space at the identity $T_{\mathrm{id}} D$ and the flow $\phi(x, 1)$ is defined as the Lie group exponential $\phi(x, 1)=\exp (\mathbf{v})$. Due to the Baker-Campbell-Hausdorff (BCH) formula [27], v can be computed directly in the log-domain by minizing the energy functional 


$$
E\left(\mathbf{v}, \mathbf{v}_{u}\right)=\frac{\left\|T-S \circ \exp \left(\mathbf{v}_{u}\right)\right\|_{L_{2}}^{2}}{\sigma_{i}^{2}}+\frac{\left\|\mathbf{v}-\mathbf{v}_{u}\right\|_{V}}{\sigma_{x}^{2}}+\frac{\|\nabla \mathbf{v}\|_{V}^{2}}{\sigma_{s}^{2}}
$$

where $\sigma_{i}, \sigma_{x}$ and $\sigma_{s}$ are parameters related to image noise, matching uncertainty and spatial smoothness respecitely, whereas $\exp \left(\mathbf{v}_{u}\right)$ is an unregularized correspondence field between $T$ and $S^{1}$.

\subsection{Enforcing temporal consistency with limited image information}

Lack of image information between time-points in homogeneous regions and tangentially to image gradients can lead to spurious deformations that adversly affect the overall optimality of the resulting deformation $\Phi_{1 N}=\phi_{1} \circ \phi_{2} \circ \cdots \circ \phi_{N-1}$. We propose a solution to this problem by means of a temporal smoothing prior defined locally in time. This enables us to define a spatial weighting of the prior based on the local model residual, thereby retaining important deformation cues from the underlying images.

Temporal Smoothing Prior A velocity field $\overline{\mathbf{v}}$ can be used as a prior to control regularization in the LogDemons registration [24] by replacing the update field $\mathbf{v}_{u}$ in the regularization of Eq. (2) by

$$
\frac{\sigma_{x}^{2} \mathbf{v}_{u}+\sigma_{t}^{2} \overline{\mathbf{v}}}{\sigma_{x}^{2}+\sigma_{t}^{2}}
$$

where $\sigma_{t}$ defines the weight of the prior. In [24], the prior field $\overline{\mathbf{v}}$ was obtained from a series of registrations between observations $S_{t_{n}}(x), n=2, \ldots, N$ to the baseline $S_{1}(x)$ by fitting a linear model over $t$ to the sequence velocity fields $\mathbf{v}_{t}(x)$ at every $x$.

When considering quickly changing morphologies, registration of all images to a common reference can be difficult and a linear model might be too restrictive. Instead, we enforce temporal smoothness by transporting the SVF $\mathbf{v}_{t-1}$ betwen $S_{t-1}$ and $S_{t}$ to the space of $S_{t}$ as $\tilde{\mathbf{v}}_{t}\left(x^{\prime}\right)=\mathbf{v}_{t-1}\left(\phi_{t-1}(x)\right), x^{\prime}=\phi_{t-1}(x)$. This corresponds to imposing constant velocity/no acceleration prior at every point of the deformation field.

Spatially Adaptive Prior The update step (3) assumes the same amount of temporal consistency over the whole image domain $\Omega$. However, this assumption is often violated due to the complex nature of biological processes. We therefore propose to weigh the influence of the temporal smoothness prior depending on the registration error at the previous time-step.

The residual at time-point $t$ between measured image $S_{t}$ and expected image $\tilde{S}_{t}=S_{t-1}\left(\phi_{t-1}\right)$ stems from the fact that the necessary spatial regularization

${ }^{1}$ without loss of generality, the intensities of all images are assumed to be scaled to $[0,1]$ 
during optimization of (2) outweighs the available image information. Mismatching regions lack the information that could drive an image-based deformation model. They should thus be subjected to stronger temporal consistency. This leads us to propose the spatially adaptive temporal smoothing prior

$$
\mathbf{p}_{t}=\frac{\sigma_{x}^{2}\left(1-\left\|S_{t}-\tilde{S}_{t}\right\|\right) \mathbf{v}_{u}+\sigma_{t}^{2}\left\|S_{t}-\tilde{S}_{t}\right\| \tilde{\mathbf{v}}_{t}}{\sigma_{x}^{2}+\sigma_{t}^{2}}
$$

Figure 2 gives an illustration of the proposed adaptive regulartization.

\section{Experiments}

We perform two sets of experiments to validate the proposed method: first on two sets of simplified synthetic models of cortical folding and secondly on a publicly available dataset of human brain development. We show that the proposed method is capable of accurately representing the deformation in all cases and results in smoother deformation fields than simple pairwise registration. We further show that using a spatio-temporal prior results in deformation models that faithfully model continuous developmental processes by evaluating its reconstruction error on unseen data.

In all experiments, the parameters of (2) and (4) are set to $\sigma_{i}, \sigma_{x}, \sigma_{s}=1$, $\sigma_{t}=.5$.

\subsection{Synthetic cortical folding}

We generate two sets of synthetic cortical folding sequences from two parametric models containing gray and white matter (Figure 3). The models represent the formation of a single respectively two sulci. We generate 20 such sequences of dimension consisting of 20 images of dimension $128 \times 128$ each, starting from a common reference shape with varying symmetry of the gyrification, the endpoints of which are shown in Figure 4.

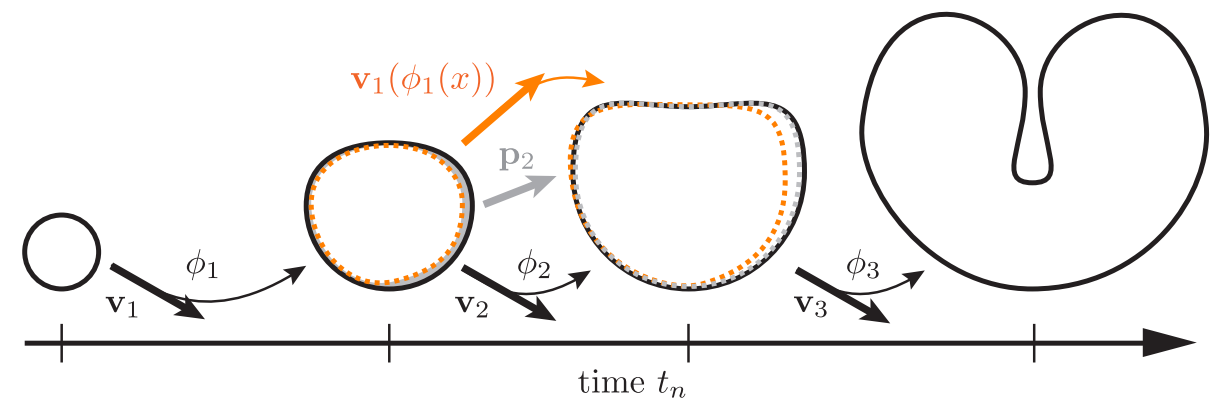

Fig. 2: Sketch of the computation of the spatially adaptive prior $\mathbf{p}_{2}$. The residual between $\tilde{S}_{t_{3}}$ (orange) and $S_{t_{3}}$ is indicated in gray. 


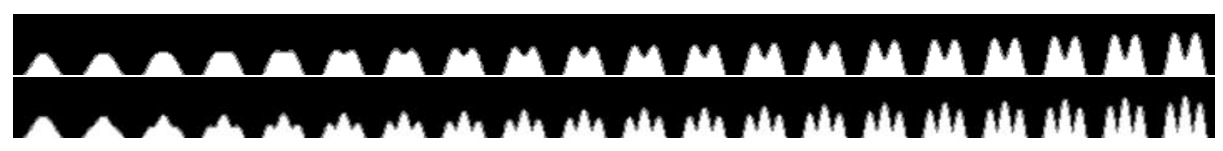

Fig. 3: Synthetic cortical folding sequences

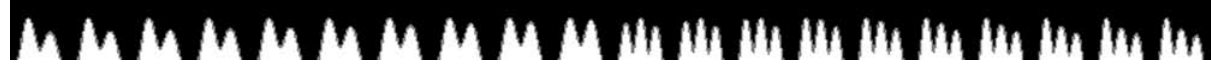

Fig. 4: Endpoints of anisotropic synthetic cortical folding sequences

Accuracy and shape of deformations In order to assess the accuracy of our method, we compute the residual between the observed $S_{t}(x), t \in(1 \ldots 20)$ and the images $S_{1}\left(\phi_{1} \circ \cdots \circ \phi_{t}(x)\right)$ obtained by deforming $S_{1}$ to the corresponding time-point. We compare concatenation of independent pair-wise registrations ${ }^{3}$ to those obtained using the proposed uniform (3) and locally adaptive (4) temporal consistency priors.

In all synthetic experiments, nearest neighbour interpolation is used to compute the deformed images $S\left(\phi_{t}(x)\right)$. The registration error is thus given as count of mislabeled pixels. Results for the single-sulcus and two-sulci experiments are shown in Figure 5 as box-plots over all anisotropic gyrification sequences and summarized as averages over all experiments in Table 1.

In both the single-sulcus (Figure 5a) and two-sulci (Figure 5b) experiments, both simple temporal smoothing and the spatially adaptive prior outperform the naive pair-wise registration. The adaptive method outperforms the uniform prior in the first frames of the simulated sequences, whereas the performance of the uniform prior is better overall.

We use the norm of the gradients of the Jacobi Determinant of the velocity fields $\left\|\nabla \operatorname{det}\left(\mathbf{v}_{t}(x)\right)\right\|, x \in C$ in order to assess spurious motion of the obtained deformations. Figure 6 shows the results for the two sets of synthetic experiments. Surprisingly, the spatially adaptive methods gives the smoothest overall deformation in both cases. The effect of accounting for temporal coherence in the serial registrations is noticeable, especially in the second half of the pairwise sulcification experiment by the strong reduction of outliers in motion complexity. The spatially uniform temporal regularization on the other hand does not reduce the overall motion complexity.

Time-point interpolation We evaluate the capacity of the deformations computed using the proposed models to interpolate between time-points. For this, we remove every second time-point from the synthetic series before computing the

\footnotetext{
${ }^{3}$ In practice, direct use of the composition of registrations computed from consecutive images lead to the propagation of matching errors resulting from finite image resolution and optimization time. We therefore initialize every registration between $S_{1}\left(\phi_{1} \circ \phi_{2} \circ \cdots \circ \phi_{t-1}\right)$ and $S_{t}$ with that between $S_{t-1}$ and $S_{t}$. Note that this does not correspond to registering all $S_{t}$ to $S_{1}$.
} 


\begin{tabular}{|l|r|r|r|r|r|r|}
\cline { 2 - 7 } \multicolumn{1}{c|}{} & \multicolumn{3}{c|}{ Dense Sampling } & \multicolumn{3}{c|}{ Interpolation } \\
\cline { 2 - 7 } \multicolumn{1}{c|}{} & pair-wise & uniform & adaptive & pair-wise & uniform & adaptive \\
\hline Single Sulcus & & & & & & \\
Mean Error & 23.83 & $\mathbf{1 9 . 2 5}$ & 22.95 & 86.64 & 134.14 & $\mathbf{8 4 . 9 4}$ \\
Smoothness & 40.06 & 41.27 & $\mathbf{2 8 . 5 6}$ & 68.35 & 71.56 & $\mathbf{5 5 . 3 4}$ \\
\hline Two Sulci & & & & & & \\
Mean Error & 40.45 & $\mathbf{2 3 . 1 3}$ & 37.56 & 128.92 & 130.90 & $\mathbf{1 0 8 . 3 0}$ \\
Smoothness & 57.05 & 42.64 & $\mathbf{3 9 . 6 9}$ & 119.73 & 111.94 & $\mathbf{1 1 1 . 3 3}$ \\
\hline
\end{tabular}

Table 1: Results of the proposed and reference methods when registering two full synthetic time-series (Dense Sampling) and reconstructing unseen time-points from sparsely sampled time-seris (Interpolation).

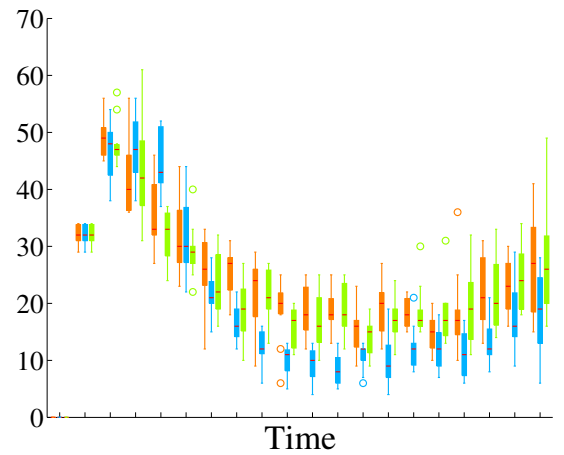

(a) Single Sulcification

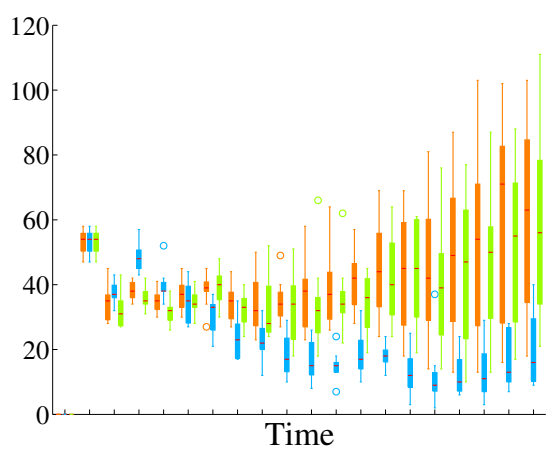

(b) Pairwise Sulcification

Fig. 5: Registration error using full image data (orange: no, blue: uniform, green: spatially adaptive temporal smoothing)

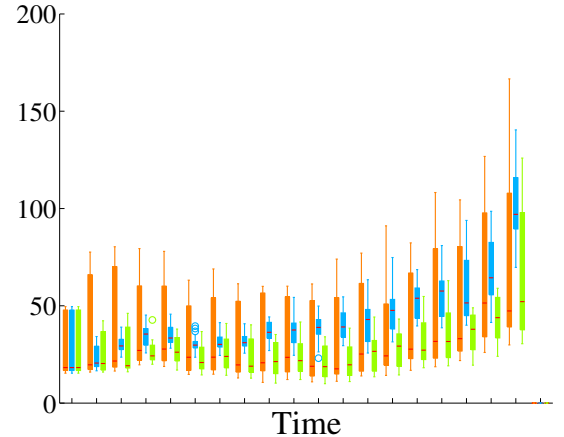

(a) Single Sulcification

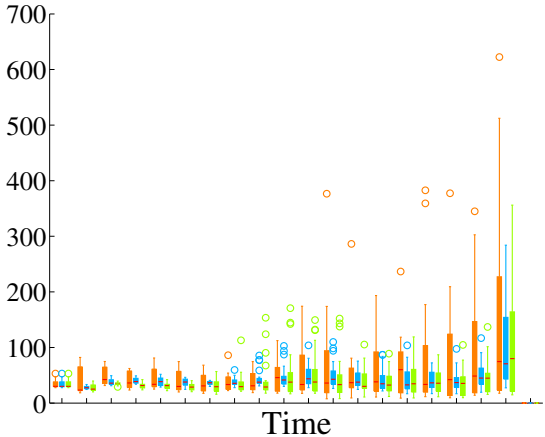

(b) Pairwise Sulcification

Fig. 6: Smoothness of deformation $\left\|\nabla \operatorname{det}\left(\mathbf{v}_{t}(x)\right)\right\|, x \in C$ (orange: no, blue: uniform, green: spatially adaptive temporal smoothing) 
serial registrations. We then scale each velocity field $\mathbf{v}_{t}$ by a factor $\frac{1}{2}$ to account for the larger gaps between observations and compute the residual to the complete series. The resulting registration errors are shown in Figure 7. Interpolated time-points are indicated on the $\mathrm{x}$-axis.

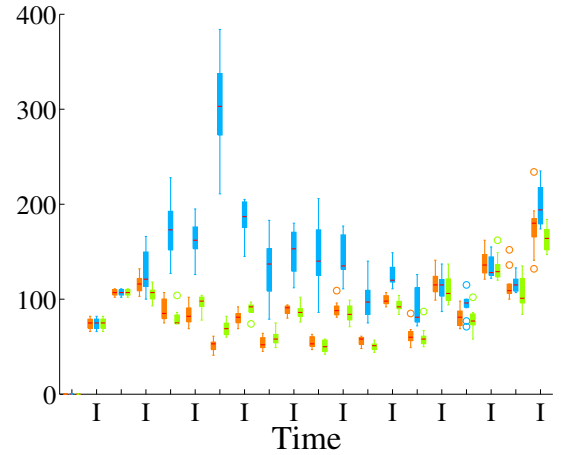

(a) Single Sulcification

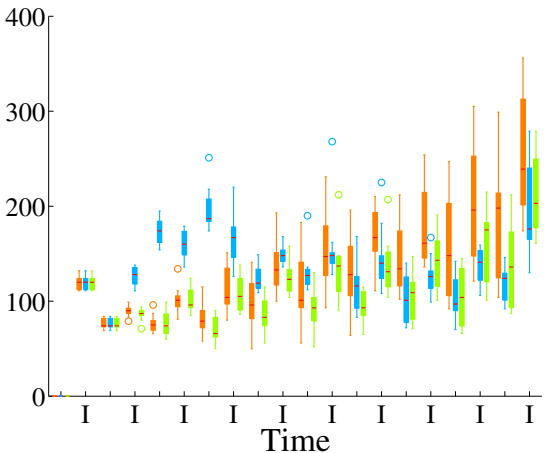

(b) Pairwise Sulcification

Fig. 7: Registration Error using half the image data (orange: no, blue: uniform, green: spatially adaptive temporal smoothing)

While the registration error is higher than when using the full series, the results show that the spatially adaptive temporal smoothing prior enables a more accurate prediction of the missing images in both experiments. Spatially uniform smoothing on the other hand leads to worse performance during most of the single-sulcification and the first half of the pair-wise sulcification experiments. We attribute this to oversmoothing at the beginning of sulcification, where its effect is not yet strongly visible (Figure 3 ). While not completely avoiding it, the spatially adaptive method however reduces this effect. This yields results that are comparable to the naive method in the first experiments and a decrease in registration error in the second. Interestingly, the registration error could also be reduced for those time-points that have been used for registration.

\subsection{Fetal brain development}

Based on the publicly available atlas [25], we compute a model of brain development in the human fetus between Gestational Weeks (GW) 23 and 35. As in the synthetic case, we evaluate the registration error and smoothness of the resulting overall deformation. In order to render the registration robust to intensity changes during development due to tissue maturation, we optimize the Residual Complexity image metric proposed in [28]. As in the synthetic experiments, the reconstruction errors are reported as Sum of Squared Differences (SSD).

The proposed spatially adaptive temporal smoothing prior yields the most accurate model in terms of reconstruction error (Table 2). Similar effects as in 


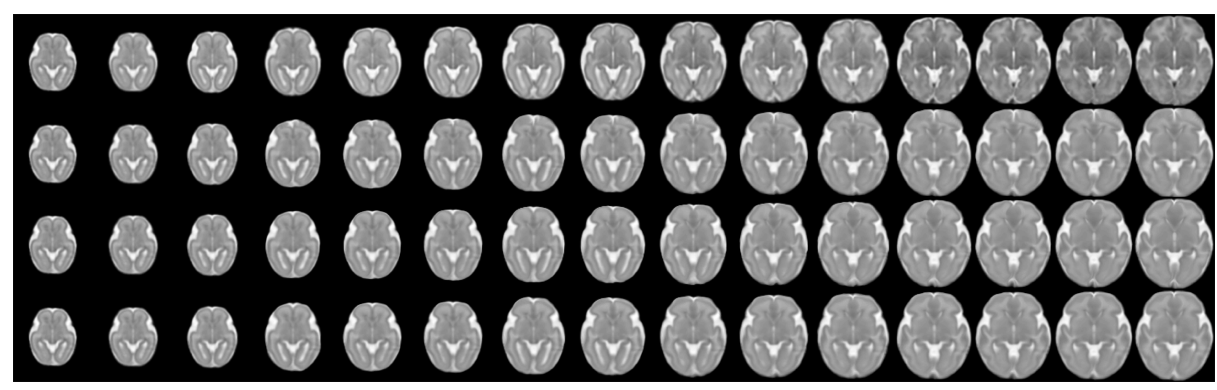

Fig. 8: Axial slices of fetal brain development seqencences between 23rd and 35th gestational week. From top to bottom no smoothing, spatially uniform temporal smoothing and spatially adaptive temporal smoothing

the synthetic case can be observed. During the first frames of the sequence, the uniform prior oversmooths the data, whereas the locally adaptive method decreases the reconstuction error (Figure 9).

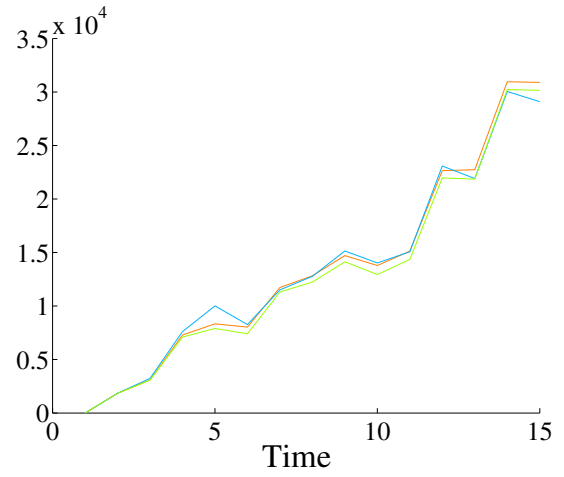

(a) Reconstruction error

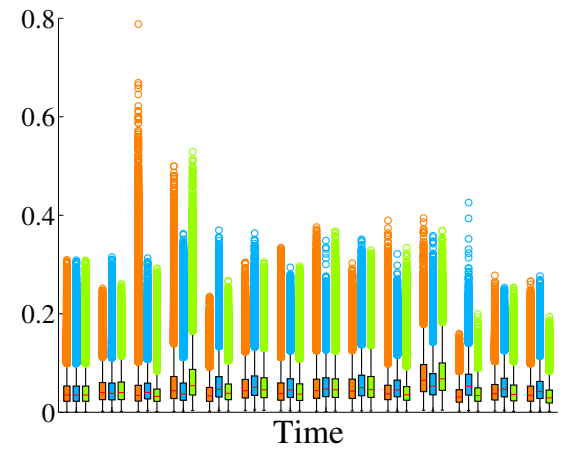

(b) Smoothness of deformation

Fig. 9: Registration Error and Deformation Smoothness on Fetal Data (orange: no, blue: uniform, green: spatially adaptive temporal smoothing)

Observing the prefrontal cortex in the last frame of the image series in detail (Figure 10) shows how the pair-wise registration fails in this region. Either temporal smoothing prior alleviates this problem. However, the spatially adaptive prior is able to account for a more complex growth pattern in the frontal part of the medial longitudinal fissure. 


\begin{tabular}{|l|r|r|r|}
\cline { 2 - 4 } \multicolumn{1}{c|}{} & pair-wise & uniform & adaptive \\
\hline Mean Error & $1.36 \times 10^{4}$ & $1.36 \times 10^{4}$ & $\mathbf{1 . 3 1} \times \mathbf{1 0}^{4}$ \\
Smoothness & $\mathbf{6 . 1 2} \times \mathbf{1 0}^{\mathbf{3}}$ & $6.72 \times 10^{3}$ & $6.2 \times 10^{3}$ \\
\hline
\end{tabular}

Table 2: Registration results on fetal brain development dataset. The proposed method yields lowest SSD and comparably smooth deformation fields.

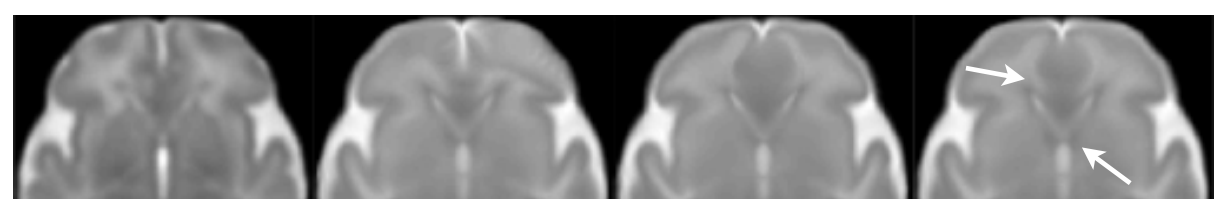

Fig. 10: Detail of (from left to right) target image, no, spatially uniform and spatially adaptive temporal smoothing. Arrows indicate exemplary regions where oversmoothing is reduced by spatially adaptive temporal smoothing.

\section{Discussion}

We have presented a method for temporal smoothing of consecutive image registrations in the framework of diffeomorphisms parametrized by stationary velocity fields. This has enabled us to define a spatially adaptive temporal smoothing prior that enables the consideration of image information while at simultaneously resulting in smoother overall deformations. We have successfully evaluated the proposed methods on synthetic image sequences of simplified cortical folding. By building deformation models on subsampled datasets, we were able to show that the temporal smoothing accurately reflects the underlying deformation. We could replicate these results on a dataset of fetal brain development, yielding a decrease in registration error and sharper image detail.

In its simplest formulation presented in this paper, the proposed method can be employed online with only the additional computational cost of just one interpolation step compared to sequential pair-wise registrations. In cases where all imaging time-points are available a-priori, more complex forward-backward smoothing schemes can be envisioned. However, propagating deformation priors further than one time-step in either direction require to enforce parallelism in the construction [29] and lead to an increase in complexity and thus susceptibility to registration error. Depending on the nature of the observed process, the selection of an initial time-point for the smoothing is likely to not only yield further increases in modeling accuracy but also deepen the understanding of the underlying dynamics. Evaluation of these effects, notably in the context of brain growth, will be the focus of future work. 


\section{References}

1. Scott, J.A., Habas, P.A., Kim, K., Rajagopalan, V., Hamzelou, K.S., CorbettDetig, J.M., Barkovich, A.J., Glenn, O.A., Studholme, C.: International Journal of Developmental Neuroscience. International Journal of Developmental Neuroscience 29(5) (2011) 529-536

2. Weiner, M.W., Veitch, D.P., Aisen, P.S., Beckett, L.A., Cairns, N.J., Green, R.C., Harvey, D., Jack, C.R., Jagust, W., Liu, E., Morris, J.C., Petersen, R.C., Saykin, A.J., Schmidt, M.E., Shaw, L., Siuciak, J.A., Soares, H., Toga, A.W., Trojanowski, J.Q., Initiative, A.D.N.: The Alzheimer's Disease Neuroimaging Initiative: A review of papers published since its inception. Alzheimer's \& Dementia 8(S) (2012) S1-S68

3. Rajagopalan, V., Scott, J., Habas, P.A., Kim, K., Corbett-Detig, J., Rousseau, F., Barkovich, A.J., Glenn, O.A., Studholme, C.: Local Tissue Growth Patterns Underlying Normal Fetal Human Brain Gyrification Quantified In Utero. Journal of Neuroscience 31(8) (2011) 2878-2887

4. Dittrich, E., Raviv, T.R., Kasprian, G., Donner, R., Brugger, P.C., Prayer, D., Langs, G.: A spatio-temporal latent atlas for semi-supervised learning of fetal brain segmentations and morphological age estimation. Medical Image Analysis 18(1) (2014) 9-21

5. Liu, W., Ribeiro, E.: A survey on image-based continuum-body motion estimation. Image and Vision Computing 29(8) (2011) 509-523

6. Bauer, M., Bruveris, M., Michor, P.W.: Overview of the Geometries of Shape Spaces and Diffeomorphism Groups. arXiv.org (2013)

7. Vialard, F.X., Risser, L., Rueckert, D., Cotter, C.J.: Diffeomorphic 3D Image Registration via Geodesic Shooting Using an Efficient Adjoint Calculation. International Journal of Computer Vision 97(2) (2011) 229-241

8. Niethammer, M., Huang, Y., Vialard, F.X.: Geodesic regression for image timeseries. In: MICCAI'11: Proceedings of the 14th international conference on Medical image computing and computer-assisted intervention. (2011) 655-662

9. Hong, Y., Shi, Y., Styner, M., Sanchez, M., Niethammer, M.: Simple geodesic regression for image time-series. In: WBIR'12: Proceedings of the 5th international conference on Biomedical Image Registration. (2012) 11-20

10. Thomas Fletcher, P.: Geodesic Regression and the Theory of Least Squares on Riemannian Manifolds. International Journal of Computer Vision 105(2) (2012) 171-185

11. Singh, N., Hinkle, J., Joshi, S., Fletcher, P.T.: A vector momenta formulation of diffeomorphisms for improved geodesic regression and atlas construction. In: Proceedings of the 2013 IEEE 10th International Symposium on Biomedical Imaging (ISBI). (2013) 1219-1222

12. Fishbaugh, J., Durrleman, S., Gerig, G.: Estimation of smooth growth trajectories with controlled acceleration from time series shape data. In: MICCAI'11: Proceedings of the 14th international conference on Medical image computing and computer-assisted intervention. (2011) 401-408

13. Beg, M., Miller, M., Trouvé, A., Younes, L.: Computing large deformation metric mappings via geodesic flows of diffeomorphisms. International Journal of Computer Vision 61(2) (2005) 139-157

14. Trouvé, A., Vialard, F.X.: A second-order model for time-dependent data interpolation: Splines on shape spaces. Quaterly of Applied Mathematics 70(2) (2012) 219-251 
15. Davis, B.C., Fletcher, P.T., Bullitt, E., Joshi, S.: Population Shape Regression From Random Design Data. In: Proc. of IEEE 11th International Conference on Computer Vision, 2007. (2007) 1-7

16. De Craene, M., Piella, G., Duchateau, N., Silva, E., Doltra, A., Gao, H., D’hooge, J., Camara, O., Brugada, J., Sitges, M.: Temporal diffeomorphic free-form deformation for strain quantification in 3D-US images. In: MICCAI'10: Proceedings of the 13th international conference on Medical image computing and computerassisted intervention. (2010) 1-8

17. Reuter, M., Schmansky, N.J., Rosas, H.D., Fischl, B.: Within-subject template estimation for unbiased longitudinal image analysis. NeuroImage 61(4) (2012) 1402-1418

18. Guizard, N., Fonov, V.S., García-Lorenzo, D., Aubert-Broche, B., Eskildsen, S.F., Collins, D.L.: Spatio-temporal regularization for longitudinal registration to an unbiased 3d individual template. In: STIA'12: Proceedings of the Second international conference on Spatio-temporal Image Analysis for Longitudinal and TimeSeries Image Data. (2012) 1-12

19. Wu, G., Wang, Q., Shen, D., Initiative, t.A.D.N.: Registration of longitudinal brain image sequences with implicit template and spatial-temporal heuristics. NeuroImage 59(1) (2012) 404-421

20. Arsigny, V., Commowick, O., Pennec, X., Ayache, N.: A log-euclidean framework for statistics on diffeomorphisms. In: MICCAI'06: Proceedings of the 9th international conference on Medical image computing and computer-assisted intervention. (2006) 924-931

21. Zhang, Z., Sahn, D., Song, X.: Frame to Frame Diffeomorphic Motion Analysis from Echocardiographic Sequences. In: Proceedings of the Third International Workshop on Mathematical Foundations of Computational Anatomy - Geometrical and Statistical Methods for Modelling Biological Shape Variability. (2011) 15-24

22. Hinkle, J., Muralidharan, P., Fletcher, P.T., Joshi, S.: Polynomial Regression on Riemannian Manifolds. In: European Conference on Computer Vision ECCV 2012. (2012) 1-14

23. Fishbaugh, J., Prastawa, M., Gerig, G., Durrleman, S.: Geodesic shape regression in the framework of currents. Information Processing in Medical Imaging (2013) $718-729$

24. Lorenzi, M., Ayache, N., Frisoni, G., Pennec, X.: 4D registration of serial brain's MR images: a robust measure of changes applied to Alzheimer's disease. (2010) $1-12$

25. Serag, A., Aljabar, P., Ball, G., Counsell, S.J., Boardman, J.P., Rutherford, M.A., Edwards, A.D., Hajnal, J.V., Rueckert, D.: Construction of a consistent highdefinition spatio-temporal atlas of the developing brain using adaptive kernel regression. NeuroImage 59(3) (2012) 2255-2265

26. Vercauteren, T., Pennec, X., Perchant, A., Ayache, N.: Diffeomorphic demons: Efficient non-parametric image registration. NeuroImage 45(1) (2009) S61-S72

27. Bossa, M., Hernandez, M., Olmos, S.: Contributions to 3D diffeomorphic atlas estimation: application to brain images. In: MICCAI'07: Proceedings of the 10th international conference on Medical image computing and computer-assisted intervention. (2007) 667-674

28. Myronenko, A., Song, X.: Intensity-based Image Registration by Minimizing Residual Complexity. IEEE Transactions on Medical Imaging (99) (2010) 1882-1891

29. Lorenzi, M., Pennec, X.: Efficient parallel transport of deformations in time series of images: from Schild's to pole ladder. Journal of Mathematical Imaging and Vision (2013) 1-13 